\title{
Influence of laser contrast on high-order harmonic generation from solid-density plasma surfaces
}

\author{
Jian Gao (高健) ${ }^{1,2}$, Feng Liu (刘峰) ${ }^{1,2, *}$, Xulei Ge (葛绪雷) ${ }^{1,2,3}$, Yanqing Deng (邓彦卿) ${ }^{1,2,4}$, \\ Guobo Zhang (张国博) ${ }^{1,2,4}$, Yuan Fang (方远) 1,2, Wenqing Wei (魏文青) ${ }^{1,2}$, Su Yang (杨骕) ${ }^{1,2}$, \\ Xiaohui Yuan (远晓辉) ${ }^{1,2}$, Min Chen (陈民) ${ }^{1,2}$, Zhengming Sheng (盛政明) ${ }^{1,2,5}$, and Jie Zhang
} (张杰) ${ }^{1,2, *}$

${ }^{1}$ Key Laboratory for Laser Plasmas (Ministry of Education) and Department of Physics and Astronomy, Shanghai Jiao Tong University, Shanghai 200240, China

${ }^{2}$ Collaborative Innovation Center of IFSA (CICIFSA), Shanghai Jiao Tong University, Shanghai 200240, China

${ }^{3}$ State Key Laboratory of Surface Physics and Department of Physics, Fudan University, Shanghai 200433, China

${ }^{4}$ College of Science, National University of Defense Technology, Changsha 410073, China

${ }^{5}$ SUPA, Department of Physics, University of Strathclyde, Glasgow G4 ONG, UK

*Corresponding author: liuf001@sjtu.edu.cn, jzhang1@sjtu.edu.cn;

The influence of the laser temporal contrast on the high-order harmonic generation from intense laser interactions with solid-density plasma surfaces is experimentally studied. A switchable plasma mirror system is set up to improve the contrast by two orders of magnitude at $10 \mathrm{ps}$ prior to the main peak. By using the plasma mirror and tuning the prepulse, the dependence of $\mathrm{HOHG}$ on laser contrast is investigated. Harmonics up to the $21^{\text {st }}$ order via the mechanism of coherent wake emission are observed only when the targets are irradiated by high contrast laser pulses by applying the plasma mirror.

Key words: temporal contrast, solid-density plasma surfaces, high-order harmonics, plasma mirror

High-order harmonic generation (HOHG) from the interactions of relativistically intense laser pulses with solid-density plasma surfaces has been demonstrated as a promising bright coherent $\mathrm{x}$-ray source in the last decade. In contrast with the harmonic generation from laser-gas interactions, whose pulse intensities are limited by gas ionization threshold and poor phase matching at laser intensities beyond $10^{15} \mathrm{~W} / \mathrm{cm}^{2[1-4]}$, the HOHG from solid targets has no limitation on laser intensities, so it can reach much higher pulse intensities to improve the conversion efficiency. Recently it is further extended to the generation of intense isolated attosecond pulses ${ }^{[5,6]}$ with near diffraction-limited spatial beam quality [7], which can be regarded as a powerful tool for diagnosing the properties of plasmas ${ }^{[8,9]}$ and imaging science ${ }^{[10,11]}$.

Since HOHG from solid targets was observed with $\mathrm{CO}_{2}$ laser for the first time by Carman et al. in $1981^{[12]}$, it has been extensively investigated both experimentally ${ }^{[13-16]}$ and theoretically ${ }^{[17-22]}$. Until now, there are two well recognized mechanisms of HOHG from plasma surfaces distinguished by the laser intensities, coherent wake emission (CWE) ${ }^{[17-19]}$ and relativistically oscillating mirror (ROM) ${ }^{[20-22]}$. Harmonics of the CWE dominate the emission at moderate laser intensities $\mathrm{a}_{0}<1$, where $a_{0}=\mathrm{e} E$ / $\mathrm{m} \omega_{\mathrm{L}} \mathrm{c}$ is the normalized vector potential, e and $\mathrm{m}$ are the electron charge and mass, $\mathrm{E}$ and $\omega_{\mathrm{L}}$ are the amplitude and frequency of laser electric field, $\mathrm{c}$ is the light velocity in vacuum. The Brunel electrons ${ }^{[23]}$ are pulled out and pushed back into overdense plasmas periodically by the driven laser and excite plasma oscillations. These oscillations coherently emit harmonics in the specular direction of the incident laser. In the CWE mechanism, the highest harmonic order $\mathrm{H}_{\max }$ is restricted by the maximum electron density of the plasma $n_{\max }$ with $H_{\max }=\sqrt{n_{\max } / n_{c}}$, where $n_{c}$ is the critical density. For the $800 \mathrm{~nm}$ laser, the $\mathrm{H}_{\max }$ with different targets of $\mathrm{CH}\left(\mathrm{n}_{\max }=225 \mathrm{n}_{\mathrm{c}}\right)$, fused silica $\left(\mathrm{n}_{\max }=400 \mathrm{n}_{\mathrm{c}}\right)$ and aluminum $\left(\mathrm{n}_{\max }=441 \mathrm{n}_{\mathrm{c}}\right)$ are 15,20 and 21 , respectively. At the relativistic laser intensities $\left(\mathrm{a}_{0} \geq 1\right)$, the ROM mechanism dominates the radiation. In the ROM mechanism, the surface of the overdense plasma is driven to oscillate with moving velocity $v \approx \mathrm{c}$ as a plasma mirror. When the incident laser is reflected by this relativistically oscillating surface, harmonics are generated due to the Doppler shift effect. Baeva et al. ${ }^{[22]}$ showed that the harmonics have a spectrum with a roll off as $\mathrm{I}_{\mathrm{H}}=\mathrm{H}^{-8 / 3}$, where $\mathrm{I}_{\mathrm{H}}$ and $\mathrm{H}$ are the intensity and the order of a given harmonic. The harmonic spectrum extends to the cut-off order $\mathrm{H}_{\max } \sim 8^{1 / 2} \gamma_{\max }{ }^{3}$, where $\gamma_{\max }$ is the maximal $\gamma$ factor of the plasma surface, determined by the laser intensities.

Both in the CWE and the ROM processes, a very steep density gradient at plasma-vacuum interface is needed for the efficient harmonic generation. The density profile at the plasma surface of a solid target depends on the laser intensities and temporal contrast, so the temporal contrast of the incident laser pulse plays a crucial role in the HOHG. In this paper, the influence of the driven laser contrast on the HOHG by CWE mechanism is investigated experimentally by using a plasma mirror system to modify the laser contrast.

The experiment was carried out using the 200 
TW Ti:sapphire laser system at the Laboratory for Laser Plasmas in Shanghai Jiao Tong University. The laser system can deliver laser pulses at $800 \mathrm{~nm}$ with energies on the target up to $5 \mathrm{~J}$, pulse durations (full width at half maximum, FWHM) of $25 \mathrm{fs}$ and repetition rates of $10 \mathrm{~Hz}$.

The sketch of experimental setup for the HOHG measurement is presented in Fig. 1. The laser beam with energy of $1.7 \mathrm{~J}$ was used in the experiment and focused by an F/4 off-axis parabolic (OAP) mirror at an incidence angle of $15^{\circ}$ to the target normal. The focal spot diameter was $6 \mu \mathrm{m}$ (FWHM) with $25 \%$ energy enclosed, giving a calculated peak intensity of $6 \times 10^{19} \mathrm{~W} / \mathrm{cm}^{2}$. Polished fused silica plates with surface flatness of $\lambda / 8$ at $632.8 \mathrm{~nm}$ were used as targets. The high-order harmonic radiations generated at specular direction were collected by a gold toroidal mirror $(\mathrm{f}=490 \mathrm{~mm})$ to the entrance slit of a flat-field spectrometer (HORIBA JOBIN YVON, TGS300) . The spectrometer consisted of a gold coated toroidal grating and a back-illuminated $\mathrm{x}$-ray charge coupled device (CCD) camera (Andor, Newton 940) . An Al filter between the slit and the grating was used to block the reflected laser beam and transmit the harmonics in the spectral range from $17.1 \mathrm{~nm}$ to 80 nm.

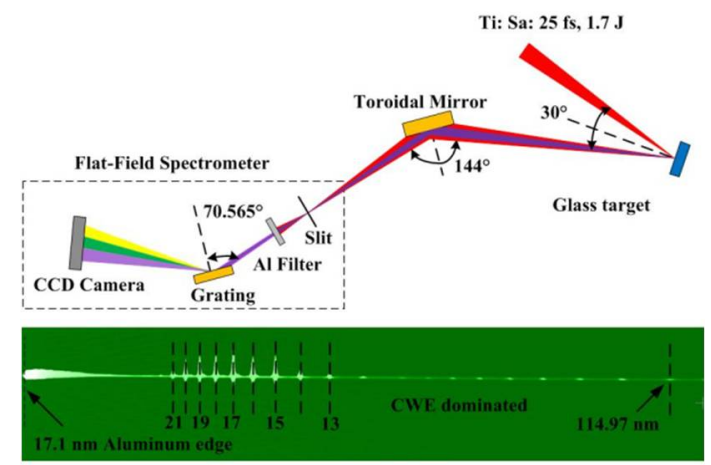

Fig. 1. The top panel is the experimental setup for HOHG. The bottom panel is the raw image of harmonic spectrum obtained from the glass target with a plasma mirror.

The HOHG process is very sensitive to the plasma density gradient at the plasma surface, which is created by the amplified spontaneous emission (ASE) and leading edge of the laser pulse. Therefore, high contrast laser pulses are preferred for the HOHG. A single plasma mirror (PM) system had been set up to enhance the laser temporal contrast. The schematic of the PM system and the measured ASE contrasts are shown in Fig. 2. As shown in Fig. 2 (a), the p-polarized laser pulse was focused by an $F / 10$ off-axis parabolic (OAP 1) mirror at an incidence angle of $10^{\circ}$ onto an anti-reflection coated fused silica glass. The silica glass was mounted on a three dimensional motorized stage, so it can provide a flesh surface for each shot. The laser intensity on the PM was properly set so that the ASE was low enough to transmit through the PM, while the leading edge of the main pulse was intense enough to ionize the PM, which could reflect the main pulse as a mirror. The reflected pulse was re-collimated by another identical off-axis parabolic (OAP 2) mirror, which was further reflected by a reflective mirror 1 (RM 1$)$ to the target chamber. The reflective mirror 2 (RM 2) could be moved into the beam path to bypass the PM system, providing laser pulses with lower contrast. The picosecond ASE contrasts were measured with a commercial third-order cross-correlator (Sequoia, Amplitude Technologies). Figure 2 (b) depicts the ASE contrasts measured with and without the PM. It is shown that the contrast at $10 \mathrm{ps}$ prior to the main peak is improved 100 fold from $\sim 10^{-8}$ to $\sim 10^{-10}$ when the PM is used. The focused laser intensity on target decreases to $3.5 \times 10^{19} \mathrm{~W} / \mathrm{cm}^{2}$, when the PM reflectivity of $58 \%$ is taken into account.
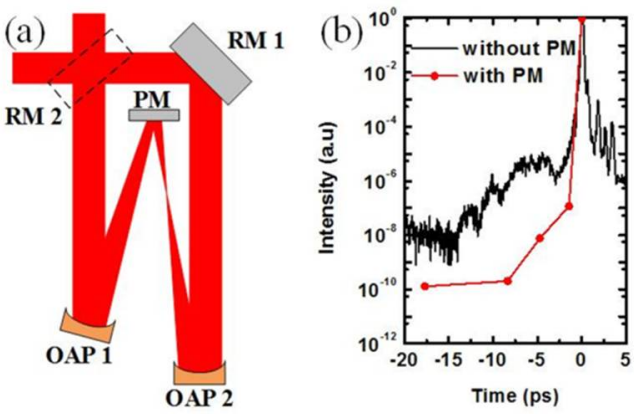

Fig. 2. (a) The schematic of the plasma mirror system. (b) The picosecond ASE contrast ratios measured without (black) and with the plasma mirror (red)

The bottom panel in Fig. 1 shows a typical raw spectrum image of harmonics obtained by a single shot when the PM is used. The intensities of harmonics from $13^{\text {rd }}$ to $21^{\text {st }}$ strongly saturate the CCD camera due to a very thin $\mathrm{Al}$ filter with thickness of $250 \mathrm{~nm}$ is used for this shot, but no harmonic with order higher than $21^{\text {st }}$ is observed. This is the characteristics of the harmonics generated by the CWE mechanism, whose highest harmonic order is limited by the maximum plasma density. In order to obtain the unsaturated spectra of harmonics, another piece of $\mathrm{Al}$ filter with thickness of $1.5 \mu \mathrm{m}$ was put in the spectrometer to attenuate the intensities on the CCD. The spectra obtained with and without PM are compared in Fig. 3. In Fig. 3(a), discrete harmonics from $17^{\text {th }}$ to $21^{\text {st }}$ orders and $\mathrm{Al} L$ absorption edge at $17.1 \mathrm{~nm}$ are clearly observed when PM is used. Figure 3(b) shows a spectrum obtained when the PM is not used by moving in RM 2 to bypass the PM. No harmonic but only plasma emission is obtained when PM is not used. The first, second and third order diffractions of $\mathrm{Al} L$ absorption edge can be seen clearly corresponding to the wavelength of $17.1 \mathrm{~nm}$, $34.2 \mathrm{~nm}$ and $51.3 \mathrm{~nm}$, respectively. The normalized intensities of the spectra in Fig. 3 are calculated by taking into account the reflectance of the toroidal mirror and the grating, the transmittance of the $\mathrm{Al}$ filters and the quantum efficiency of the CCD camera. 

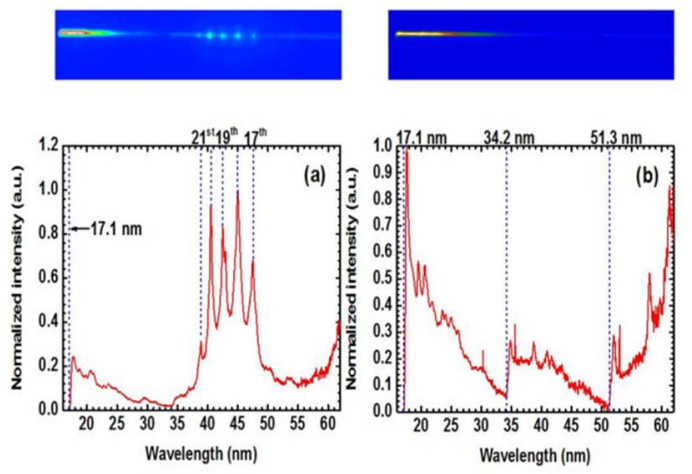

Fig. 3. (a) The raw spectral image (top panel) and spectrum of high-order harmonics (bottom panel) obtained with (a) and without (b) plasma mirror in specular direction of the incident laser.
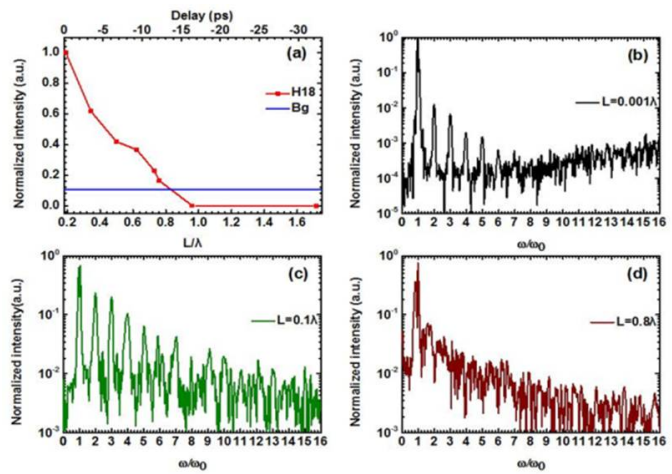

Fig. 4. (a) The dependence of $18^{\text {th }}$ harmonic intensities on plasma scale lengths $L$ obtained in the experiment (red). Blue line indicates the intensity of background plasma emission. The harmonic spectra obtained by PIC simulations with different plasma scale lengths (b) $L=0.001 \lambda$ (black), (c) $L=0.1 \lambda$ (green) and (d) $L=0.8 \lambda$ (brown).

In the experiment, no harmonic with order over CWE cutoff at $21^{\text {st }}$ is observed. There are three possible reasons for the ROM harmonics not generated, including the intensities, the temporal contrast and the incidence angle of the laser. First, the normalized vector potential $\mathrm{a}_{0}$ is the key parameter determining whether the harmonics are generated by the CWE or the ROM mechanism. The CWE harmonics dominate the radiation of non-relativistic laser intensities with $\mathrm{a}_{0}<1$, while the ROM harmonics dominate the radiation of relativistic laser intensities with $\mathrm{a}_{0} \geq 1$. However, the peak laser intensity when the PM is used in the experiment is $3.5 \times 10^{19} \mathrm{~W} / \mathrm{cm}^{2}$ with corresponding $\mathrm{a}_{0}=4$, which is beyond the relativistic intensity threshold. Second, it is verified both by numerical simulations ${ }^{[17}$, ${ }^{24]}$ and experiments ${ }^{[25,26]}$ that there is an optimum plasma scale length range $L$ for the CWE or the ROM harmonic generation. When the plasma density is steplike $L \sim 0$, both the CWE and the ROM could not be efficiently excited. The ROM requires a low density plasma at the plasma surface with $0.1 \lambda_{\text {laser }}<L<\lambda_{\text {laser. }}$. On the other hand, the CWE requires much steeper plasma with $0<L<0.1 \lambda_{\text {laser }}$. In order to change $L$, we introduce a prepulse with pulse duration (FWHM) of $50 \mathrm{fs}$ and intensity of $2 \times 10^{16} \mathrm{~W} / \mathrm{cm}^{2} . L$ could be continuously tuned by varying the relative delay between the prepulse and the driven pulse from -33 ps to 3 ps. No ROM harmonic is observed, while the CWE harmonic intensities are found to decrease with increasing the delay until they disappear after delay of $-12.33 \mathrm{ps}$. We use the hydrodynamic code MULTI to estimate $L$ at different prepulse delay. The experimental data of $18^{\text {th }}$ harmonic intensities dependence on $L$ are presented in Fig. 4(a). 2D particle-in-cell (PIC) simulations are also performed to obtain the HHG spectra with three typical $L$ as shown in Fig. 4(b-d), where a p-polarized laser field with intensity of $3.5 \times 10^{19}$ W/ $/ \mathrm{cm}^{2}\left(\mathrm{a}_{0}=4\right)$ impinges an overdense plasma with maximum plasma electron density $400 n_{c}$ with an incidence angle of $15^{\circ}$. From the simulation results, it can be clearly seen in Fig. 4(b) that the harmonic generation is not efficient for $L=0.001 \lambda_{\text {laser }}$. The harmonic intensities are enhanced dramatically up to $L=0.1 \lambda_{\text {laser }}$ in Fig. 4(c). However, if the scale length is further increased to $L=0.8 \lambda_{\text {laser }}$, no harmonics but only plasma emission could be seen in Fig. 4(d). In CWE mechanism, the wakefield cannot radiate efficiently if the plasma gradient is very steep $(L \sim 0)^{[17,24,27]}$. On the other hand, the Brunel electrons which excite wakefield inside the plasma, cannot be generated if preplama is too long [24, 27]. Harmonic generation efficiency first increases very fast and then decreases slowly when $L$ is increased, which agrees with the simulation results shown in Fig. 4(b-d). In experiments, the laser leading edge always produces preplasma before the laser peak arriving at the target surface. Harmonic intensities show a monotonic decrease with the increase of $L$ as shown in Fig 4(a). According to the above discussion, the laser intensities and contrast are ruled out for no ROM harmonic generation of our experiment. Finally, it is shown in Ref. 27 that the efficiency of HOHG reaches its maximum at optimal incident angle of $55^{\circ}$. In our experiment, the incidence angle of the laser is $15^{\circ}$, which is much smaller than the optimal angle of $55^{\circ}$. In the future, we will increase the incidence angle to investigate its impact on the HOHG.

In summary, we find that in our experiment the temporal contrast of the incident laser is crucial for HOHG from plasma surfaces. A PM system is used to improve the ASE contrast of the laser by two orders of magnitude from $\sim 10^{-8}$ to $\sim 10^{-10}$ at $10 \mathrm{ps}$ prior to the main peak. The harmonics up to the $21^{\text {st }}$ order are observed from the solid plasma surface of the plain glass when the PM system is adopted, which is generated by the CWE mechanism. No harmonic except plasma emission is obtained when the PM system is not used.

This work is supported by National Basic Research Program of China (Grant No. 2013CBA01502), National Natural Science Foundation of China (Grant Nos. 11305103, 11374209, 11374210, and 11205100), Natural Science Foundation of Shanghai (Grant No. 13ZR1456300) and International Science \& Technology Cooperation Program of China (Grant No. 2014DFG02330).

1. J. L. Krause, K. J. Schafer, and K. C. Kulander, "High-order harmonic generation from atoms and ions in the high intensity regime" , Phys. Rev. Lett. 68, 3535 (1992).

2. P. B. Corkum, "Plasma perspective on strong-field multiphoton ionization", Phys. Rev. Lett. 71, 1994 
(1993).

3. Candong Liu, Pengfei Wei, Jing Miao, Chunmei Zhang, Yansui Huang, Yinghui Zheng, Yuxin Leng, and Zhinan Zeng, "Generation of high-order harmonic continuum with two-hump-structure infrared laser pulse", Chin. Opt. Lett. 12, 030201 (2014)

4. Jiaming Jiang, Pengfei Wei, Zhinan Zeng, Xiaolong Yuan, Yinghui Zheng, Xiaochun Ge, Ruxin Li, and Zhizhan Xu, "Selection of odd or even harmonics by a multi-color laser field with macroscopic phase-matching", Chin. Opt. Lett. 13, 050202 (2015).

5. M. Yeung, J. Bierbach, E. Eckner, S. Rykovanov, S. Kuschel, A. Sävert, M. Förster, C. Rödel, G. G. Paulus, S. Cousens, M. Coughlan, B. Dromey and M. Zepf, "Noncollinear Polarization Gating of Attosecond Pulse Trains in the Relaticistic Regime", Phys. Rev. Lett. 115, 193903 (2015)

6. H. Vincenti and F. Quéré, "Attosecond Lighthouses: How To Use Spatiotemporally Coupled Light Fields To Generate Isolated Attosecond Pulses" , Phys. Rev. Lett. 108, 113904 (2012).

7. B. Dromey, D. Adams, R. Hörlein, Y. Nomura, S. G. Rykovanov, D. C. Carroll, P. S. Foster, S. Kar, K. Markey, P. McKenna, D. Neely, M. Geissler, G. D. Tsakiris and M. Zepf, "Diffraction-limited performance and focusing of high harmonics from relativistic plasmas" , Nat. Phys. 5, 146 (2009).

8. U. Wagner, M. Tatarakis, A. Gopal, F. N. Beg, E. L. Clark, A. E. Dangor, R. G. Evans, M. G. Haines, S. P. D. Mangles, P. A. Norreys, M. S. Wei, M. Zepf, and K. Krushelnick, "Laboratory measurements of 0.7 GG magnetic fields generated during high-intensity laser interactions with dense plasmas" , Phys. Rev. E. 70, 026401 (2004)

9. Malvache, A. Borot, F. Quéré, and R. Lopez-Martens, "Coherent wake emission spectroscopy as a probe of steep plasma density profiles" , Phys. Rev. E. 87, 035101 (2013)

10. R. L. Sandberg, A. Paul, D. A. Raymondson, S. Hädrich, D. M. Gaudiosi, J. Holtsnider, R. I. Tobey, O. Cohen, M. M. Murnane, and H. C. Kapteyn, "Lensless Diffractive Imaging Using Tabletop Coherent High-Harmonic Soft-X-Ray Beams”, Phys. Rev. Lett. 99, 098103 (2007).

11. Ravasio, D. Gauthier, F. R. N. C. Maia, M. Billon, J. P. Caumes, D. Garzella, M. Géléoc, O. Gobert, J. F. Hergott, A. M. Pena, H. Perez, B. Carré, E. Bourhis, J. Gierak, A. Madouri, D. Mailly, B. Schiedt, M. Fajardo, J. Gautier, P. Zeitoun, P. H. Bucksbaum, J. Hajdu, and H. Merdji, "Single-Shot Diffractive Imaging with a Table-Top Femtosecond Soft X-Ray Laser-Harmonics Source", Phys. Rev. Lett. 103, 028104 (2009).

12. R. L. Carman, D. W. Forslund, and J. M. Kindel, "Visible Harmonic Emission as a Way of Measuring Profile Steepening", Phys. Rev. Lett. 46, 29 (1981)

13. P. A. Norreys, M. Zepf, S. Moustaizis, A. P. Fews, J. Zhang, P. Lee, M. Bakarezos, C. N. Danson, A. Dyson, P. Gibbon, P. Loukakos, D. Neely, F. N. Walsh, J. S. Wark, and A. E. Dangor, "Efficient Extreme UV Harmonics Generated from Picosecond Laser Pulse Interactions with Solid Targets", Phys. Rev. Lett. 76, 1932 (1996)

14. J. Zhang, M. Zepf, P. A. Norreys, A. E. Dangor, M. Bakarezos, C. N. Danson, A. Dyson, A. P. Fews, P. Gibbon, M. H. Key, P. Lee, P. Loukakos, S. Moustaizis, D. Neely, F. N. Walsh, and J. S. Wark, "Coherence and bandwidth measurements of harmonics generated from solid surfaces irradiated by intense picoseconds laser pulses", Phys. Rev. A, 54, 1597 (1996).
15. B. Dromey, M. Zepf, A. Gopal, K. Lancaster, M. S. Wei, K. Krushelnick, M. Tatarakis, N. Vakakis, S. Moustaizis, R. Kodama, M. Tampo, C. Stoeckl, R. Clarke, H. Habara, D. Neely, S. Karsch and P. Norreys, "High harmonic generation in the relativistic limit" , Nat. Phys. 2, 456 (2006).

16. M. Bocoum, M. Thévenet, F. Böhle, B. Beaurepaire, A. Vernier, A. Jullien, J. Faure, and R. Lopez-Martens, "Anticorrelated Emission of High Harmonics and Fast Electron Beams From Plasma Mirrors", Phys. Rev. Lett. 116, 185001 (2016)

17. B. Dromey, S. G. Rykovanov, D. Adams, R. Hörlein, Y. Nomura, D. C. Carroll, P. S. Foster, S. Kar, K. Markey, P. McKenna, D. Neely, M. Geissler, G. D. Tsakiris, and M. Zepf, “Tunable Enhancement of High Harmonic Emission from Laser Solid Interactions", Phys. Rev. Lett. 102, 225002 (2009)

18. B. Bezzerides, R. D. Jones, and D. W. Forslund, "Plasma Mechanism for Ultraviolet Harmonic Radiation Due to Intense $\mathrm{CO}_{2}$ Light", Phys. Rev. Lett. 49, 202 (1982).

19. F. Quéré, C. Thaury, P. Monot, S. Dobosz, and Ph. Martin, "Coherent Wake Emission of High-Order Harmonics from Overdense Plasma”, Phys. Rev. Lett. 96, 125004 (2006).

20. S. V. Bulanov, N. M. Naumova, and F. Pegoraro, "Interaction of an ultrashort, relativistically strong laser pulse with an overdense plasma", Phys. Plasmas 1, 745 (1994).

21. R. Lichters, J. Meyer-ter-Vehn, and A. Pukhov, "Short - pulse laser harmonics from oscillating plasma surfaces driven at relativistic intensity", Phys. Plasmas 3 3425 (1996)

22. T. Baeva, S. Gordienko, and A. Pukhov, "Theory of high-order harmonic generation in relativistic laser interaction with overdense plasma", Phys. Rev. E. 74, 046404 (2006).

23. F. Brunel, "Not-So-Resonant, Resonant Absorption" , Phys. Rev. Lett. 59, 52 (1987).

24. Tarasevitch, K. Lobov, C. Wünsche, and D. von der. Linde, "Transition to the Relativistic Regime in High Order Harmonic Generation", Phys. Rev. Lett. 98, 103902 (2007)

25. Rödel, D. an der Brügge, J. Bierbach, M. Yeung, T. Hahn, B. Dromey, S. Herzer, S. Fuchs, A. G. Pour, E. Eckner, M. Behmke, M. Cerchez, O. Jäckel, D. Hemmers, T. Toncian, M. C. Kaluza, A. Belyanin, G. Pretzler, O. Willi, A. Pukhov, M. Zepf, and G. G. Paulus, "Harmonic Generation from Relativistic Plasma Surfaces in Ultrasteep Plasma Density Gradients", Phys. Rev. Lett. 109, $125002(2012)$

26. S. Kahaly, S. Monchocé, H. Vincenti, T. Dzelzainis, B. Dromey, M. Zepf, Ph. Martin, and F. Quéré, "Direct Observation of Density-Gradient Effects in Harmonic Generation from Plasma Mirrors", Phys. Rev. Lett. 110, 175001 (2013).

27. C. Thaury and F. Quéré, "High-order harmonic and attosecond pulse generation on plasma mirrors: basic mechanisms", J. Phys. B 43, 213001 (2010). 